\title{
Synthesis of monoazo disperse dyes, their dyeing performance on polyester fibers and antimicrobial activity
}

\author{
Sandip K. Patel ${ }^{\mathrm{a}}$, Pratixa K. Patel ${ }^{\mathrm{b}}$, G. M. Malik ${ }^{\mathrm{a}, *}$ \\ ${ }^{a}$ Department of Chemistry, Navyug Science College, Surat-395009, Gujarat, India. \\ ${ }^{b}$ Department of Chemistry, Narmada College of Science and Commerce Zadeshwar, Bharuch-392011 Gujarat, \\ India.
}

\begin{abstract}
Different monoazo disperse dyes $N$-(4-nitrophenyl)-2-((4-(3'-nitro phenyl)-1, 3-thiazol-2-yl) amino)acetamide derivatives has been synthesized in the current study using different diazotized aryl amines. These were characterized using elemental analysis, ${ }^{I} H$ NMR and IR spectra. Their dyeing performance on polyester fiber was assessed and fastness properties of these dyes were evaluated by applying them to polyester fiber. These dyes showed moderate anti-bacterial and anti-fungal activities.
\end{abstract}

Keywords: 2-Amino-4-(3'-nitro phenyl) thiazole, chloroacetylchloride, 4-nitro aniline, dyeing performance, ${ }^{1} H$ NMR, IR Spectra, antimicrobial activity.

\section{Introduction}

Disperse dyes are organic colorants and have less water solubility which are applied in colloidal dispersions to hydrophobic textile fibers to produce desired colour. There has been significant increase in the development of disperse dyes due to increasing use of polyester fibers and their blend [1-3], 2-amino thiazole derivatives [4-8] have been utilized as heterocyclic components since long for many disperse dyes. The synthesis of thiazole, 2-amino-4-phenyl thiazole and their derivatives has got significant importance because a large number of natural products and drugs comprise of this heterocyclic moiety [9-11]. It was our main objective to synthesize monoazo disperse dyes based on 2-chloro-N-(4-nitrophenyl)acetamide [12,13]. The characteristic data of synthesized dyes and dyeing properties also studied [14-18]. The newly synthesized monoazo disperse dyes exhibited moderate to fairly good light, washing and sublimation fastness. The synthesized dyes sample were screened for antibacterial activity against Staphylococcus aureus, Escherichia coli, Bacillus subtilis and Pseudomonas aeruginosa and antifungal activity against candida albicans [19-24].

\section{Materials and Methods}

All reagents were of analytical reagent grade and were used without further purification. All the synthesized compounds were characterized by their spectral analysis. The melting points of the compounds were determined using Stuart smp 10 melting point apparatus and are uncorrected. The purity of dyes was determined by thin- layer chromatography (TLC) using silica gel-G coated Al-plates and spots were visualized under uv radiation. IR spectra were recorded on FTIR spectrophotometer Perkin Elmer RX-1 using $\mathrm{KBr}$ pellets. ${ }^{1} \mathrm{H}$ NMR spectra obtained on Bruker Avance-II 400 NMR spectrometer using DMSO solvent and TMS as internal reference (chemical shifts in $\delta$, ppm). Elemental analysis carried on Perkin Elmer (USA) 2400 series instrument. The synthesized dyes were screened for their antimicrobial activity using the Kirby-Bauer method. All the compounds were screened for their in vitro antimicrobial activity against bacterial strains Escherichia coli, Pseudomonas aeruginosa, Staphylococcus aureus, Bacillus subtilis and fungi Candida albicans at $50 \mu \mathrm{g} / \mathrm{ml}$ concentration and Ciprofloxacin and Flucanazole using as standards respectively. The fastness to light, wash and sublimation was assessed in accordance with ISO 105. A convenient laboratory method was used for dyeing polyester to employ high temperature $\left(130^{\circ} \mathrm{C}\right)$ and high pressure (24-30psi.). The dye bath exhaustion (\%E) of the dyed fiber was determined according to the method.

\section{Preparation of 2-amino-4-(3'-Nitro phenyl) thiazole (1)}

In $250 \mathrm{ml}$ R.B.F mixture of ( $16.5 \mathrm{~g}, 0.1 \mathrm{~mole}) \mathrm{m}$-nitro acetophenone and thiourea (15.2 g, $0.2 \mathrm{~mole})$ was added. Then bromine ( $23.97 \mathrm{~g}, 0.15 \mathrm{~mole}$ ) drop wise added, after addition of bromine the reaction mixture was heated in water bath for 8-10 hours. It was cooled and content was washed with water. The residue left was dissolved in boiling water and filtered. The filtrate was made alkaline by addition of liquor ammonia. The solid (1) thus obtained was filtered, dried and recrystalized from ethanol.

Yield $81 \%$, M.P.: $185-188{ }^{\circ} \mathrm{C}$ IR ( $\mathrm{KBr} \mathrm{cm}^{-1)} 3444$ (N-H str.), 3113 (C-H str.), 1512 ( $\mathrm{NO}_{2}$ str.), 713 (C-S str.), 1339 (C-N str.). 


\section{Preparation of 2-chloro-N-(4-nitrophenyl)acetamide(2)}

4-nitro aniline ( $2.76 \mathrm{~g}, 0.02$ mole) and $15 \mathrm{ml}$ glacial acetic acid was added to a $250 \mathrm{ml}$ R.B.F. then cooled to $0-5{ }^{\circ} \mathrm{C}$. Chloroacetyl chloride $(3.39 \mathrm{ml}, 0.02$ mole) was slowly added to R.B.F. with continuous stirring. When addition was complete, the reaction mixture was stirred at room temperature for 3 hours. The reaction mixture dumped into $20 \%$ sodium acetate solution. The solid (2) thus obtained was filtered washed with water, dried and recrystalized from $80 \%$ ethanol and $20 \%$ water solution. Yield: $90 \%$, M.P.: $186-188^{\circ}$ C.

\section{Preparation of N-(4-nitrophenyl)-2-((4-(3'-nitro phenyl)-1, 3-thiazol-2-yl) amino) acetamide (3)}

In a $250 \mathrm{ml}$ R.B.F. 2-amino-4-(3'-nitro phenyl) thiazole) $(2.21 \mathrm{~g}, 0.01 \mathrm{~mole})(1)$ and 2-chloro-N-(4nitrophenyl)acetamide $\left(2.14 \mathrm{~g}, 0.01\right.$ mole) (2) in $30 \mathrm{ml}$ glacial acetic acid was taken, then slowly $\mathrm{K}_{2} \mathrm{CO}_{3}(6.9 \mathrm{~g}$, 0.05 mole) was added into reaction mixture. When addition was completed, the reaction mixture was heated for 6 hours at $85-90{ }^{\circ} \mathrm{C}$. The reaction mixture dumped into $20 \%$ sodium acetate solution. The solid (3) thus obtained was filtered washed with water, dried and recrystalized from ethanol. Yield: $80 \%$, M.P.: $160-165^{\circ} \mathrm{C}$.

\section{Diazotization and coupling reaction $\left(\mathbf{S P}_{\mathbf{1}}-\mathbf{S P}_{\mathbf{1 5}}\right)$}

Aryl amines $\left(0.01\right.$ mole) dissolved in $\mathrm{HCl}(5 \mathrm{ml}, 50 \%)$ was cooled to $0-5{ }^{\circ} \mathrm{C}$ in an ice-bath. A solution of sodium nitrite $(0.01$ mole, $0.69 \mathrm{~g})$ in water $(5 \mathrm{ml})$ previously cooled to $0-5{ }^{\circ} \mathrm{C}$ was added over a period of ten minutes with continuous stirring and maintaining the temperature at $0-5{ }^{\circ} \mathrm{C}$, further the reaction mixture was stirred for an hour keeping constant positive test on starch iodide paper. The excess of nitrous acid was then destroyed using sulfamic acid. The resulting solution was used for coupling reaction.

N-(4-nitrophenyl)-2-((4-(3'-nitro phenyl)-1, 3-thiazol-2-yl) amino) acetamide (3) (0.01mole) was dissolved in glacial acetic acid $(30 \mathrm{ml})$. Then cooled below $5{ }^{\circ} \mathrm{C}$ in an ice-bath. The above mentioned diazonium chloride solution was added drop wise over a period of 10-15 minutes, maintaining the $\mathrm{pH} 7.0$ to 7.5 by simultaneous addition of aqueous sodium acetate $(10 \% \mathrm{w} / \mathrm{v})$, further the reaction mixture was stirred for 3 hours at $0-5{ }^{\circ} \mathrm{C}$. The solid dye was precipitated. The dye was filtered, washed with water to remove acid completely and dried and recrystalized from acetone.

\section{Spectral Properties of Dyes}

\section{Results And Discussion}

The visible absorption spectrum of dyes was recorded in DMF and their properties are shown in (Table 1). From (Table 1), it is clear that the value of $\lambda_{\max }$ depends on the nature and position of coupling component used.

IR spectra of synthesized dyes described in (Table 3), compound (3) showed N-H stretching vibration at $3417 \mathrm{~cm}^{-1}, \mathrm{C}-\mathrm{H}$ stretching vibration at $3115 \mathrm{~cm}^{-1}, \mathrm{C}=\mathrm{O}$ stretching vibration at $1649 \mathrm{~cm}^{-1}, \mathrm{~N}=\mathrm{N}$ stretching vibration at $1575, \mathrm{C}-\mathrm{S}-\mathrm{C}$ stretching vibration at $1471 \mathrm{~cm}^{-1}, \mathrm{C}-\mathrm{S}$ stretching in thiazole at $750 \mathrm{~cm}^{-1}, 1518 \mathrm{~cm}^{-1}$ is due to presence of $\mathrm{NO}_{2}$ group. The ${ }^{1} \mathrm{H}$ NMR of all dyes showed in (Table 3). The ${ }^{1} \mathrm{H}$ NMR spectra of all dyes were recorded in DMSO. The compound (2) showed spectra exhibited multiplets in the region at $\delta$ 7.06-8.75 ppm for 12 aromatic protons. The Methylene attached to carbonyl group showed singlet at $\delta 3.40 \mathrm{ppm}$. While presence of $-\mathrm{NH}$ in thiazole and aromatic ring showed singlet at $\delta 4.24 \mathrm{ppm}$ and $\delta 7.00 \mathrm{ppm}$, respectively.

\section{Dyeing Properties Dyeing method}

All the synthesized dyes $\mathrm{SP}_{1}-\mathrm{SP}_{15}$ were applied on polyester fabric in $2 \%$ depth by HTHP method. High temperature $\left(130{ }^{\circ} \mathrm{C}\right)$ and high pressure is a convenient method for dyeing polyester fibers in the laboratory. Glycerin-bath high temperature beaker dyeing machine was used. $10 \mathrm{ml}$ DMF was added and dispersion of the dye was produced by dissolving the appropriate amount of dye (2\% depth) and then added dropwise with continuous stirring to the dyebath (liquor ratio 20:1) containing 1\% Setamol WS (BASF) as anionic dispersing agent. The $\mathrm{pH}$ of dye solution was adjusted to 4 to 5 using aqueous acetic acid. Already wetted-out polyester fabric pieces were added. Dyeing was run by rising the dyebath temperature upto $130{ }^{\circ} \mathrm{C}$ at a rate of $2{ }^{\circ} \mathrm{C} / \mathrm{min}$, maintaining at this temperature for 1 hour, under (24-30 psi) pressure. After completion of process cooling upto $50{ }^{\circ} \mathrm{C}$ was done, the dyed fabric pieces were rinsed with cold water and reduction was cleared properly ( $1 \mathrm{~g} / 1$ sodium hydroxide, $1 \mathrm{~g} / 1$ sodium hydrosulphite, 10 minutes and $\left.80{ }^{\circ} \mathrm{C}\right)$. The dyed sample was rinsed with hot and cold water and finally the dyeing air- dried.

\section{Fastness Properties \\ Light Fastness}

The light fastness study was carried out using a xenon light fastness tester having a xenon arc lamp. The dyed sample was assessed by comparing change in colour of specimen with standard dye patterns of specific rating (1 to 8 ). The higher the rating, the better is the light fastness. The results are given in (Table 2). 


\section{Wash Fastness}

Fastness to washing was assessed using following method. The washing solution contained soap $5 \mathrm{~g} / \mathrm{l}$ and anhydrous sodium carbonate $2 \mathrm{~g} / \mathrm{l}$, washing temperature was $95^{\circ} \mathrm{C}$, the duration of washing was $30 \mathrm{~min}$, and the material to liquor ratio was 5:1. After washing, the specimen was removed and rinsed with hot water $\left(35^{\circ} \mathrm{C}\right)$ until the rinsed water showed no alkalinity. It was dried in the air. The effect of the change in colour was expressed and defined by reference to grey scale. The results are given in (Table 2).

\section{Sublimation Fastness}

The dyed fabric was stitched to undyed cotton fabric on one side and undyed polyester fabric on the other side. The composite dyeing specimens were placed in a heating device (sublimation tester) electrical heating system and kept there for $30 \mathrm{~s}$ at $180{ }^{\circ} \mathrm{C}$. The dried fabric was removed and kept in air for 4 hours. The effect of dyed fabrics on the cotton and polyester fabrics was measured and assessed with grey scales. The results are given in (Table 2).

As shown in (Table 2), the light fastness of each dye is rated as 3-5 for polyester, which shows light fastness is moderate to good. The wash fastness of each dye is rated as 3-5 for polyester showing wash fastness is good to excellent. The sublimation fastness of each dye is rated as 3-5 for polyester, which also shows rubbing fastness is good to excellent.

\section{Antimicrobial Activity}

Antibacterial and antifungal activities of synthesized compounds were examined in vitro by KirbyBauer disc diffusion method. The zone of inhibition $(\mathrm{mm})$ was determined using disc diffusion method according to the standard procedure at concentrations $50 \mu \mathrm{g} / \mathrm{ml}$. All the compounds were tested for activity against Staphylococcus aureus, Escherichia coli, Bacillus subtilis, Pseudomonas aeruginosa and Candida albicans. Ciprofloxacin and Flucanazole were employed as the standard (Table 4).

\section{Conclusion}

All the synthesized monoazo disperse dyes based on 2-chloro-N-(4-nitrophenyl)acetamide synthesized by conventional methods and their different properties were studied applying them to polyester fabric. The dyes showed good dyeing performance on polyester fibers. The dyes gave generally yellow, orange, brown, red, and cream shades. Table 2 shows moderate to fairly good light fastness. Dyes $\mathrm{SP}_{1}, \mathrm{SP}_{4}, \mathrm{SP}_{5}, \mathrm{SP}_{7}, \mathrm{SP}_{9}, \mathrm{SP}_{12}$, and $\mathrm{SP}_{15}$ showed better light fastness. The wash-fastness of all the dyes was also of very good order. All the samples showed moderate to fairly good antibacterial activity against Escherichia coli, Pseudomonas aeruginosa, Staphylococcus aureus and Bacillus subtilis. All the samples showed moderate to fairly good antifungal activity against Candida albicans. Standard drugs Ciprofloxacin and Flucanazole were used for the comparison purpose. The results are shown in (Table 4).

\section{Acknowledgment}

Authors are thankful to the Principal, Navyug Science College, Surat and Principal, V. S. Patel College of Arts \& Science, Bilimora, for laboratory facilities and Atul Limited, for dyeing and computer colour matching facilities.

\section{References}

[1]. B. R. Modi, D. M. Vashi and K. R. Desai, Ind. J. of Chem. Technology, Sept., (1994) 317-318.

[2]. D. W. Rangnekar, V. R. Kanetkar, G. S. Shankarling and J. V. Malanker, J. Heterocycl. Chem., 36 (1999) 95

[3]. D.W. Rangnekar and D. D. Rajadhyaksha, Ind. J. Textile Res., 16 (1991) 170.

[4]. Hari Raghav Maradiya and Vithal Soma Patel, Bulletin of the Chemists and Technologists of Macedonia. 21 (1) (2002) 57-64.

[5]. Kamaljit Singh, Sarbjit Singh, John A.Taylor, Dyes and Pigments. 54 (2002) 189-200.

[6]. PC. Miranda, LM. Rodrigues, MST. Goncalves, SPG. Costa, R. Hardina and AMF. Oliveira, Advance in Colour Science and Technology. 4 (2001) 1 .

[7]. M. A. Metwally, E. Abdel-Latif, F. A. Amer, G. Kaupp, Dyes and Pigments. 60 (2004) 249-264

[8]. J. S. Yadav, B.V. Subba Reddy, Rao Y. Gopala, A.V. Narsaiah, Tetrahedron Letters. 49 (2008) $2381-2383$.

[9]. R. M. Dodson and L. Carroll King, J Am chem. Soc., 67 (1945) 2242

[10]. D. P. Bhoot, R. C. Khunt, V. K. Shankhavara, and H. H. Parekh, J. Sci., Islamic Republic of Iran. 17(4), (2006) 323.

[11]. K. F. Ansari. and C. Lal, J. Chem. Sci. 121 (2009) 1017.

[12]. S. K. Zadafiya, and G.M. Malik, Fibers and Polymers. 14 (6) (2013) 904-908.

[13]. S. K. Zadafiya, J. H. Tailor. and G. M. Malik, J. of Chem. http://dx.doi.org10.1155/2013/ Article ID 851418, 5 pages.

[14]. M. M. Dalal and K. R. Desai, Oriental J. Chem. 11 (1995) 71.

[15]. Hari Raghav Maradiya and Vithal Soma Patel. Fibers and Polymers. 2 (3) (2001) 153-158.

[16]. Hari R. Maradiya, J. Serb. Chem. Soc. 67 (11) (2002) 709-718.

[17]. Divyesh R Patel and Keshav C Patel. Fibers and Polymers. 11 (4) (2010) 537-544.

[18]. Nikhil M Parekh and Kalpana C Maheria, Fibers and Polymers. 13 (7) (2010) 880-886. 
Synthesis of monoazo disperse dyes, their dyeing performance on polyester fibers and .....

[19]. G. Saravanan, V Alagarsamy, T. G. V Pavitra, G. Chanukyakumar, Y. Savithri, L. Naresh and Avinah P. International J. Pharma and Biosciences. 1 (2010) 1-8.

[20]. Deepak Singh., Srivastava Manish., A. K. Gyananchandran and P. D. Gokulan., Journal of Current Pharmaceuticals Research. 4 (2010) 16-19.

[21]. A. K. Prajapati and Vishal. P. Modi, J. Chil. Chem. Soc. 55 (2) (2010) 240-243.

[22]. S. R. Partan, N. S. Dighe, S. A. Nirmal, A. N. Merekar, R. B. Laware, H. V. Shinde, D. S. Musmade, Asian J. Research Chem. 2(2) (2009) 196-201.

[23]. G. Saravanan., V. Alagarsamy., C. R.Prakash, Selvam., T. Panneer., V. Karthick and P. dinesh kumar, Rasayan J. Chem. 2 (3) (2009) 746-752.

[24]. P. P. Bhosale, R. S. Chavan and A.V. Bhosale, Indian J. Chem. 51B (2012) 1649-1654.

\section{Reaction scheme:}

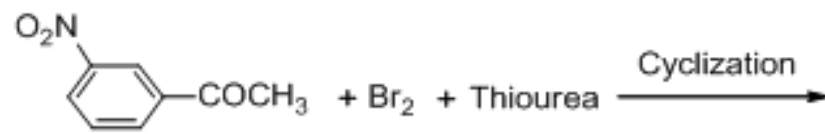<smiles>Nc1ccc([N+](=O)[O-])cc1</smiles>
$\mathrm{ClCH}_{2} \mathrm{COCl}$

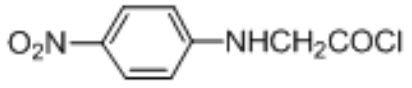

(2)<smiles></smiles>

(1)<smiles>Nc1nc(-c2cccc([N+](=O)[O-])c2)cs1</smiles>

(1)

Condensation<smiles>O=C(CNc1ccc([N+](=O)[O-])cc1)Nc1nc(-c2cccc([N+](=O)[O-])c2)cs1</smiles><smiles>[R]c1ccc(N=Nc2sc(NC(=O)CNc3ccc([N+](=O)[O-])cc3)nc2-c2cccc([R])c2)cc1</smiles>

Where, $\mathrm{R}=\mathrm{H}, \mathrm{NO}_{2}, \mathrm{CH}_{3}, \mathrm{Cl}, \mathrm{F}, \mathrm{OCH}_{3}, \mathrm{OH}, \mathrm{NHCOCH}_{3}$. 
Synthesis of monoazo disperse dyes, their dyeing performance on polyester fibers and .....

Table 1- Physical properties of $\mathrm{SP}_{1}-\mathrm{SP}_{15}$ Dyes

\begin{tabular}{|c|c|c|c|c|c|c|c|c|}
\hline \multirow[b]{2}{*}{$\begin{array}{l}\text { Dye } \\
\text { No. }\end{array}$} & \multirow[b]{2}{*}{$\mathbf{R}$} & \multirow[b]{2}{*}{$\begin{array}{l}\text { Molecular } \\
\text { formula }\end{array}$} & \multirow[b]{2}{*}{$\begin{array}{l}\text { Molecular } \\
\text { weight } \\
\mathrm{g} / \mathrm{mole}\end{array}$} & \multirow[b]{2}{*}{$\begin{array}{c}\text { Yield } \\
\%\end{array}$} & \multirow[b]{2}{*}{$\underset{\mathrm{C}}{\text { M.P. }}$} & \multirow[b]{2}{*}{$\begin{array}{l}\lambda_{\max } \\
(\mathrm{nm})\end{array}$} & \multicolumn{2}{|c|}{ Nitrogen $(\%)$} \\
\hline & & & & & & & Found & Calcd. \\
\hline $\mathrm{SP}_{1}$ & $\mathrm{H}-$ & $\mathrm{C}_{23} \mathrm{H}_{17} \mathrm{~N}_{7} \mathrm{O}_{5} \mathrm{~S}$ & 503 & 80 & 146 & 466 & 19.35 & 19.43 \\
\hline $\mathrm{SP}_{2}$ & $3-\mathrm{NO}_{2-}$ & $\mathrm{C}_{23} \mathrm{H}_{16} \mathrm{~N}_{8} \mathrm{O}_{7} \mathrm{~S}$ & 548 & 79 & 238 & 490 & 19.48 & 20.43 \\
\hline $\mathrm{SP}_{3}$ & $4-\mathrm{NO}_{2-}$ & $\mathrm{C}_{23} \mathrm{H}_{16} \mathrm{~N}_{8} \mathrm{O}_{7} \mathrm{~S}$ & 548 & 81 & 250 & 508 & 20.52 & 20.43 \\
\hline $\mathrm{SP}_{4}$ & $3-\mathrm{CH}_{3}-$ & $\mathrm{C}_{24} \mathrm{H}_{19} \mathrm{~N}_{7} \mathrm{O}_{5} \mathrm{~S}$ & 517 & 74 & 146 & 450 & 18.90 & 18.95 \\
\hline $\mathrm{SP}_{5}$ & $4-\mathrm{CH}_{3}$ & $\mathrm{C}_{24} \mathrm{H}_{19} \mathrm{~N}_{7} \mathrm{O}_{5} \mathrm{~S}$ & 517 & 76 & 140 & 460 & 18.82 & 18.95 \\
\hline $\mathrm{SP}_{6}$ & 3-Cl- & $\mathrm{C}_{23} \mathrm{H}_{16} \mathrm{ClN}_{7} \mathrm{O}_{5} \mathrm{~S}$ & 537 & 80 & 188 & 466 & 18.34 & 18.23 \\
\hline $\mathrm{SP}_{7}$ & 4-Cl- & $\mathrm{C}_{23} \mathrm{H}_{16} \mathrm{ClN}_{7} \mathrm{O}_{5} \mathrm{~S}$ & 537 & 75 & 172 & 467 & 18.12 & 18.23 \\
\hline $\mathrm{SP}_{8}$ & 4-F- & $\mathrm{C}_{23} \mathrm{H}_{16} \mathrm{FN}_{7} \mathrm{O}_{5} \mathrm{~S}$ & 521 & 78 & 144 & 454 & 18.76 & 18.80 \\
\hline $\mathrm{SP}_{9}$ & $3-\mathrm{OCH}_{3-}$ & $\mathrm{C}_{24} \mathrm{H}_{19} \mathrm{~N}_{7} \mathrm{O}_{6} \mathrm{~S}$ & 533 & 75 & 150 & 450 & 18.46 & 18.38 \\
\hline $\mathrm{SP}_{10}$ & $4-\mathrm{OCH}_{3-}$ & $\mathrm{C}_{24} \mathrm{H}_{19} \mathrm{~N}_{7} \mathrm{O}_{6} \mathrm{~S}$ & 533 & 68 & 154 & 440 & 18.30 & 18.38 \\
\hline $\mathrm{SP}_{11}$ & $2,4-\left(\mathrm{NO}_{2}\right)_{2-}$ & $\mathrm{C}_{23} \mathrm{H}_{15} \mathrm{~N}_{9} \mathrm{O}_{9} \mathrm{~S}$ & 593 & 80 & 128 & 533 & 21.15 & 21.24 \\
\hline $\mathrm{SP}_{12}$ & $2-\mathrm{OH}-$ & $\mathrm{C}_{23} \mathrm{H}_{17} \mathrm{~N}_{7} \mathrm{O}_{6} \mathrm{~S}$ & 519 & 77 & 154 & 450 & 18.80 & 18.87 \\
\hline $\mathrm{SP}_{13}$ & $2-\mathrm{OH}, 4-\mathrm{NO}_{2-}^{-}$ & $\mathrm{C}_{23} \mathrm{H}_{16} \mathrm{~N}_{8} \mathrm{O}_{8} \mathrm{~S}$ & 564 & 65 & 140 & 460 & 19.76 & 19.85 \\
\hline $\mathrm{SP}_{14}$ & $2,6-(\mathrm{Cl})_{2,4}-\mathrm{NO}_{2-}$ & $\mathrm{C}_{23} \mathrm{H}_{14} \mathrm{Cl}_{2} \mathrm{~N}_{8} \mathrm{O}_{7} \mathrm{~S}$ & 617 & 79 & 142 & 490 & 18.27 & 18.15 \\
\hline $\mathrm{SP}_{15}$ & $4-\mathrm{NHCOCH}_{3-}$ & $\mathrm{C}_{25} \mathrm{H}_{20} \mathrm{~N}_{8} \mathrm{O}_{6} \mathrm{~S}$ & 560 & 63 & 146 & 476 & 20.15 & 19.99 \\
\hline
\end{tabular}

Table 2- Fastness properties of $\mathrm{SP}_{1}-\mathrm{SP}_{15}$ Dyes

\begin{tabular}{|c|c|c|c|c|c|c|c|c|c|}
\hline \multirow{2}{*}{$\begin{array}{l}\text { Dye } \\
\text { No. }\end{array}$} & \multirow{2}{*}{$\begin{array}{c}\text { Shade } \\
\text { on } \\
\text { Polyester }\end{array}$} & \multicolumn{2}{|c|}{ Fastness to } & \multicolumn{2}{|c|}{$\begin{array}{c}\text { Sublimation fastness at } \\
180 \mathrm{C}\end{array}$} & \multirow[t]{2}{*}{$\begin{array}{c}\text { Exhaustion } \\
\%\end{array}$} & \multirow[t]{2}{*}{$\begin{array}{c}\text { Fixation } \\
\%\end{array}$} & \multirow[t]{2}{*}{$\begin{array}{l}\text { K/S } \\
\text { Value }\end{array}$} & \multirow[t]{2}{*}{$\begin{array}{l}\mathbf{R} \\
\text { Value }\end{array}$} \\
\hline & & Light & Washing & $\begin{array}{l}\text { Staining of } \\
\text { cotton }\end{array}$ & $\begin{array}{r}\text { Staining of } \\
\text { Polyester }\end{array}$ & & & & \\
\hline $\mathrm{SP}_{1}$ & Yellow & 5 & 4 & 4 & 4 & 71.50 & 77.5 & 18.54 & 9.52 \\
\hline $\mathrm{SP}_{2}$ & Light yellow & 4 & 5 & 4 & 5 & 75.64 & 90.1 & 3.52 & 27.2 \\
\hline $\mathrm{SP}_{3}$ & Reddish orange & 4 & 4 & $4-5$ & 4 & 72.65 & 85.5 & 4.89 & 23.5 \\
\hline $\mathrm{SP}_{4}$ & Reddish yellow & 5 & 5 & $4-5$ & 5 & 77.50 & 87.1 & 17.46 & 10.1 \\
\hline $\mathrm{SP}_{5}$ & Turmeric yellow & 5 & $4-5$ & 4 & $4-5$ & 79.80 & 92.3 & 21.53 & 8.5 \\
\hline $\mathrm{SP}_{6}$ & Yellow & 4 & 5 & 5 & 5 & 74.55 & 89.2 & 20.28 & 8.67 \\
\hline $\mathrm{SP}_{7}$ & Dark yellow & 5 & $4-5$ & $4-5$ & 5 & 80.20 & 88.3 & 17.95 & 10.2 \\
\hline $\mathrm{SP}_{8}$ & Turmeric yellow & 4 & 4 & $4-5$ & 4 & 78.70 & 92.5 & 14.93 & 13.5 \\
\hline $\mathrm{SP}_{9}$ & Light yellow & 5 & $4-5$ & 4 & $4-5$ & 76.20 & 91.2 & 11.55 & 18.35 \\
\hline $\mathrm{SP}_{10}$ & Cream & $3-4$ & 4 & $3-4$ & 4 & 74.80 & 89.75 & 1.38 & 29.25 \\
\hline $\mathrm{SP}_{11}$ & Maroon & $4-5$ & 5 & $4-5$ & 5 & 77.90 & 91.70 & 11.45 & 13.3 \\
\hline $\mathrm{SP}_{12}$ & Cream & 5 & 5 & 5 & $4-5$ & 75.75 & 90.4 & 1.42 & 29.02 \\
\hline $\mathrm{SP}_{13}$ & Pinkish Orange & $4-5$ & 4 & $4-5$ & $3-4$ & 78.20 & 92.3 & 4.43 & 23.9 \\
\hline $\mathrm{SP}_{14}$ & Reddish pink & 4 & $4-5$ & 4 & 3 & 79.33 & 93.7 & 3.70 & 26.24 \\
\hline $\mathrm{SP}_{15}$ & Brown & 5 & 5 & $4-5$ & $4-5$ & 76.65 & 91.5 & 3.65 & 26.80 \\
\hline
\end{tabular}

Light fastness: 1- poor, 2- slight, 3- moderate, 4- fair, 5- good, 6- very good, 7- excellent Wash and sublimation fastness: 1- poor, 2- fair, 3-good, 4- very good, 5- excellent

Table 3- IR and ${ }^{1} \mathrm{H}$ NMR data of $\mathrm{SP}_{1}-\mathrm{SP}_{15}$ Dyes

\begin{tabular}{|c|c|c|}
\hline $\begin{array}{l}\text { Dye } \\
\text { No. }\end{array}$ & $\mathrm{IR}(\mathrm{KBr}) \mathrm{cm}^{-1}$ & ${ }^{1} \mathrm{H}$ NMR (DMSO) Chemical shift in $\square$ ppm \\
\hline $\mathrm{SP}_{1}$ & $\begin{array}{l}3420 \text { (N-H str.), } 3118(\mathrm{C}-\mathrm{H}), 1640(\mathrm{C}=\mathrm{O}), 1576 \\
(\mathrm{~N}=\mathrm{N}), 1520\left(\mathrm{NO}_{2}\right), 1468(\mathrm{C}-\mathrm{S}-\mathrm{C}), 760(\mathrm{C}-\mathrm{S})\end{array}$ & $\begin{array}{l}3.34\left(\mathrm{~S}, 2 \mathrm{H},-\mathrm{COCH}_{2}\right), 4.20(\mathrm{~S}, 1 \mathrm{H},-\mathrm{NH}) \\
6.98(\mathrm{~S}, 1 \mathrm{H},-\mathrm{NH}), 7.00-8.70(\mathrm{M}, 13 \mathrm{H}, \mathrm{Ar}-\mathrm{H})\end{array}$ \\
\hline $\mathrm{SP}_{2}$ & $\begin{array}{l}3410(\mathrm{~N}-\mathrm{H}), 3110(\mathrm{C}-\mathrm{H}), 1645(\mathrm{C}=\mathrm{O}), 1580(\mathrm{~N}=\mathrm{N}), \\
1524\left(\mathrm{NO}_{2}\right), 1475(\mathrm{C}-\mathrm{S}-\mathrm{C}), 755 \quad(\mathrm{C}-\mathrm{S})\end{array}$ & $\begin{array}{l}3.40\left(\mathrm{~S}, 2 \mathrm{H},-\mathrm{COCH}_{2}\right), 4.24(\mathrm{~S}, 1 \mathrm{H},-\mathrm{NH}) \\
7.00(\mathrm{~S}, 1 \mathrm{H},-\mathrm{NH}), 7.06-8.75(\mathrm{M}, 12 \mathrm{H}, \mathrm{Ar}-\mathrm{H})\end{array}$ \\
\hline $\mathrm{SP}_{3}$ & $\begin{array}{l}3417(\mathrm{~N}-\mathrm{H}), 3115(\mathrm{C}-\mathrm{H}), 1649(\mathrm{C}=\mathrm{O}), 1575(\mathrm{~N}=\mathrm{N}) \\
1518\left(\mathrm{NO}_{2}\right), 1471(\mathrm{C}-\mathrm{S}-\mathrm{C}), 750 \quad(\mathrm{C}-\mathrm{S}) \\
\end{array}$ & $\begin{array}{l}3.38\left(\mathrm{~S}, 2 \mathrm{H},-\mathrm{COCH}_{2}\right), 4.22(\mathrm{~S}, 1 \mathrm{H},-\mathrm{NH}) \\
7.10(\mathrm{~S}, 1 \mathrm{H},-\mathrm{NH}), 7.12-8.72(\mathrm{M}, 12 \mathrm{H}, \mathrm{Ar}-\mathrm{H})\end{array}$ \\
\hline $\mathrm{SP}_{4}$ & $\begin{array}{l}3417(\mathrm{~N}-\mathrm{H}), 3015\left(\mathrm{CH}_{3}\right), 3115(\mathrm{C}-\mathrm{H}), 1651(\mathrm{C}=\mathrm{O}) \\
1575(\mathrm{~N}=\mathrm{N}), 1518\left(\mathrm{NO}_{2}\right), 1480 \quad(\mathrm{C}-\mathrm{S}-\mathrm{C}), 765(\mathrm{C}-\mathrm{S})\end{array}$ & $\begin{array}{l}2.30\left(\mathrm{~S}, 3 \mathrm{H},-\mathrm{CH}_{3}\right), 3.32\left(\mathrm{~S}, 2 \mathrm{H},-\mathrm{COCH}_{2}\right), \\
4.20(\mathrm{~S}, 1 \mathrm{H},-\mathrm{NH}), 7.04(\mathrm{~S}, 1 \mathrm{H},-\mathrm{NH}), 7.08-8.70(\mathrm{M}, 12 \mathrm{H}, \\
\mathrm{Ar}-\mathrm{H})\end{array}$ \\
\hline $\mathrm{SP}_{5}$ & $\begin{array}{l}3412(\mathrm{~N}-\mathrm{H}), 3010\left(\mathrm{CH}_{3}\right), 3113(\mathrm{C}-\mathrm{H}), 1655(\mathrm{C}=\mathrm{O}), \\
1570(\mathrm{~N}=\mathrm{N}), 1518\left(\mathrm{NO}_{2}\right), 1477 \quad(\mathrm{C}-\mathrm{S}-\mathrm{C}), 760(\mathrm{C}-\mathrm{S})\end{array}$ & $\begin{array}{l}2.32\left(\mathrm{~S}, 3 \mathrm{H},-\mathrm{CH}_{3}\right), 3.34\left(\mathrm{~S}, 2 \mathrm{H},-\mathrm{COCH}_{2}\right), \\
4.22(\mathrm{~S}, 1 \mathrm{H},-\mathrm{NH}), 7.06(\mathrm{~S}, 1 \mathrm{H},-\mathrm{NH}), 7.10-8.79(\mathrm{M}, 12 \mathrm{H} \text {, } \\
\text { Ar-H) }\end{array}$ \\
\hline $\mathrm{SP}_{6}$ & $\begin{array}{l}3416(\mathrm{~N}-\mathrm{H}), 3113(\mathrm{C}-\mathrm{H}), 1658(\mathrm{C}=\mathrm{O}), 1578(\mathrm{~N}=\mathrm{N}), \\
1520\left(\mathrm{NO}_{2}\right), 1482(\mathrm{C}-\mathrm{S}-\mathrm{C}), 753 \quad(\mathrm{C}-\mathrm{S}), 728(\mathrm{C}-\mathrm{Cl})\end{array}$ & $\begin{array}{l}3.32\left(\mathrm{~S}, 2 \mathrm{H},-\mathrm{COCH}_{2}\right), 4.26(\mathrm{~S}, 1 \mathrm{H},-\mathrm{NH}) \\
6.9(\mathrm{~S}, 1 \mathrm{H},-\mathrm{NH}), 6.94-8.95(\mathrm{M}, 12 \mathrm{H}, \mathrm{Ar}-\mathrm{H}) \\
\end{array}$ \\
\hline $\mathrm{SP}_{7}$ & $\begin{array}{l}3418(\mathrm{~N}-\mathrm{H}), 3117(\mathrm{C}-\mathrm{H}), 1669(\mathrm{C}=\mathrm{O}), 1574(\mathrm{~N}=\mathrm{N}), \\
1522\left(\mathrm{NO}_{2}\right), 1474(\mathrm{C}-\mathrm{S}-\mathrm{C}), 756 \quad(\mathrm{C}-\mathrm{S}), 730(\mathrm{C}-\mathrm{Cl})\end{array}$ & $\begin{array}{l}3.36\left(\mathrm{~S}, 2 \mathrm{H},-\mathrm{COCH}_{2}\right), 4.30(\mathrm{~S}, 1 \mathrm{H},-\mathrm{NH}) \\
6.96(\mathrm{~S}, 1 \mathrm{H},-\mathrm{NH}), 7.00-8.98(\mathrm{M}, 12 \mathrm{H}, \mathrm{Ar}-\mathrm{H})\end{array}$ \\
\hline $\mathrm{SP}_{8}$ & 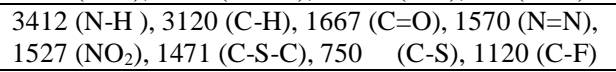 & $\begin{array}{l}3.30\left(\mathrm{~S}, 2 \mathrm{H},-\mathrm{COCH}_{2}\right), 4.25(\mathrm{~S}, 1 \mathrm{H},-\mathrm{NH}) \\
6.98(\mathrm{~S}, 1 \mathrm{H},-\mathrm{NH}), 7.08-8.86(\mathrm{M}, 12 \mathrm{H}, \mathrm{Ar}-\mathrm{H})\end{array}$ \\
\hline & $3414(\mathrm{~N}-\mathrm{H}), 3112(\mathrm{C}-\mathrm{H}), 1660(\mathrm{C}=\mathrm{O}), 1568(\mathrm{~N}=\mathrm{N})$ & $2.36\left(\mathrm{~S}, 3 \mathrm{H},-\mathrm{CH}_{3}\right), 3.32\left(\mathrm{~S}, 2 \mathrm{H},-\mathrm{COCH}_{2}\right)$ \\
\hline
\end{tabular}


Synthesis of monoazo disperse dyes, their dyeing performance on polyester fibers and .....

\begin{tabular}{|c|c|c|}
\hline $\mathrm{SP}_{9}$ & $\begin{array}{ll}1530\left(\mathrm{NO}_{2}\right), 1478(\mathrm{C}-\mathrm{S}-\mathrm{C}), 755 & (\mathrm{C}-\mathrm{S}) \\
1160\left(\mathrm{C}^{-\mathrm{OCH}_{3}}\right) & \\
\end{array}$ & $\begin{array}{l}4.28(\mathrm{~S}, 1 \mathrm{H},-\mathrm{NH}), 7.08(\mathrm{~S}, 1 \mathrm{H},-\mathrm{NH}), 7.10-8.78(\mathrm{M}, 12 \mathrm{H}, \\
\text { Ar-H) }\end{array}$ \\
\hline $\mathrm{SP}_{10}$ & $\begin{array}{l}3419(\mathrm{~N}-\mathrm{H}), 3108(\mathrm{C}-\mathrm{H}), 1670(\mathrm{C}=\mathrm{O}), 1576(\mathrm{~N}=\mathrm{N}), \\
1532\left(\mathrm{NO}_{2}\right), 1473(\mathrm{C}-\mathrm{S}-\mathrm{C}), 759 \quad(\mathrm{C}-\mathrm{S}), 1150(\mathrm{C}- \\
\left.\mathrm{OCH}_{3}\right)\end{array}$ & $\begin{array}{l}2.40\left(\mathrm{~S}, 3 \mathrm{H},-\mathrm{CH}_{3}\right), 3.36\left(\mathrm{~S}, 2 \mathrm{H},-\mathrm{COCH}_{2}\right) \\
4.30(\mathrm{~S}, 1 \mathrm{H},-\mathrm{NH}), 7.10(\mathrm{~S}, 1 \mathrm{H},-\mathrm{NH}), 7.14-8.85(\mathrm{M}, 12 \mathrm{H}, \\
\mathrm{Ar}-\mathrm{H})\end{array}$ \\
\hline $\mathrm{SP}_{11}$ & $\begin{array}{l}3412(\mathrm{~N}-\mathrm{H}), 3116(\mathrm{C}-\mathrm{H}), 1644(\mathrm{C}=\mathrm{O}), 1572(\mathrm{~N}=\mathrm{N}), \\
1516\left(\mathrm{NO}_{2}\right), 1460(\mathrm{C}-\mathrm{S}-\mathrm{C}), 754 \quad(\mathrm{C}-\mathrm{S})\end{array}$ & $\begin{array}{l}3.36\left(\mathrm{~S}, 2 \mathrm{H},-\mathrm{COCH}_{2}\right), 4.28(\mathrm{~S}, 1 \mathrm{H},-\mathrm{NH}) \\
7.15(\mathrm{~S}, 1 \mathrm{H},-\mathrm{NH}), 7.2-9.0(\mathrm{M}, 11 \mathrm{H}, \mathrm{Ar}-\mathrm{H})\end{array}$ \\
\hline $\mathrm{SP}_{12}$ & $\begin{array}{l}3414(\mathrm{~N}-\mathrm{H}), 3050(\mathrm{O}-\mathrm{H}), 3110(\mathrm{C}-\mathrm{H}), 1642(\mathrm{C}=\mathrm{O}) \\
1566(\mathrm{~N}=\mathrm{N}), 1512\left(\mathrm{NO}_{2}\right), 1456 \quad \text { (C-S-C), } 750(\mathrm{C}-\mathrm{S})\end{array}$ & $\begin{array}{l}3.32\left(\mathrm{~S}, 2 \mathrm{H},-\mathrm{COCH}_{2}\right), 4.26(\mathrm{~S}, 1 \mathrm{H},-\mathrm{NH}) \\
7.04(\mathrm{~S}, 1 \mathrm{H},-\mathrm{NH}), 5.6(\mathrm{~S}, 1 \mathrm{H},-\mathrm{OH}), 7.08-8.92 \\
(\mathrm{M}, 12 \mathrm{H}, \mathrm{Ar}-\mathrm{H})\end{array}$ \\
\hline $\mathrm{SP}_{13}$ & $\begin{array}{l}3410(\mathrm{~N}-\mathrm{H}), 3054(\mathrm{O}-\mathrm{H}), 3118(\mathrm{C}-\mathrm{H}), 1640(\mathrm{C}=\mathrm{O}), \\
1562(\mathrm{~N}=\mathrm{N}), 1514\left(\mathrm{NO}_{2}\right), 1452 \quad \text { (C-S-C), } 752(\mathrm{C}-\mathrm{S})\end{array}$ & $\begin{array}{l}3.34\left(\mathrm{~S}, 2 \mathrm{H},-\mathrm{COCH}_{2}\right), 4.28(\mathrm{~S}, 1 \mathrm{H},-\mathrm{NH}), \\
7.06(\mathrm{~S}, 1 \mathrm{H},-\mathrm{NH}), 5.64(\mathrm{~S}, 1 \mathrm{H},-\mathrm{OH}), 7.20-8.96(\mathrm{M}, 11 \mathrm{H}, \\
\text { Ar-H) }\end{array}$ \\
\hline SP & $\begin{array}{l}3408(\mathrm{~N}-\mathrm{H}), 3116(\mathrm{C}-\mathrm{H}), 1660(\mathrm{C}=\mathrm{O}), 1578(\mathrm{~N}=\mathrm{N}), \\
1512\left(\mathrm{NO}_{2}\right), 1472(\mathrm{C}-\mathrm{S}-\mathrm{C}), 764 \quad(\mathrm{C}-\mathrm{S}), 726(\mathrm{C}-\mathrm{Cl})\end{array}$ & $\begin{array}{l}3.38\left(\mathrm{~S}, 2 \mathrm{H},-\mathrm{COCH}_{2}\right), 4.22(\mathrm{~S}, 1 \mathrm{H},-\mathrm{NH}) \\
7.04(\mathrm{~S}, 1 \mathrm{H},-\mathrm{NH}), 7.10-8.94(\mathrm{M}, 10 \mathrm{H}, \mathrm{Ar}-\mathrm{H})\end{array}$ \\
\hline $\mathrm{SP}_{15}$ & $\begin{array}{l}3412(\mathrm{~N}-\mathrm{H}), 3114(\mathrm{C}-\mathrm{H}), 1662(\mathrm{C}=\mathrm{O}), 1580(\mathrm{~N}=\mathrm{N}), \\
1514\left(\mathrm{NO}_{2}\right), 1477(\mathrm{C}-\mathrm{S}-\mathrm{C}), 760 \quad(\mathrm{C}-\mathrm{S}), 1683(\mathrm{C}- \\
\left.\mathrm{NHCOCH}_{3}\right)\end{array}$ & $\begin{array}{l}2.2\left(\mathrm{~S}, 3 \mathrm{H},-\mathrm{CH}_{3}\right), 3.30\left(\mathrm{~S}, 2 \mathrm{H},-\mathrm{COCH}_{2}\right), \\
4.22(\mathrm{~S}, 1 \mathrm{H},-\mathrm{NH}), 7.12(\mathrm{~S}, 2 \mathrm{H},-\mathrm{NH}), 7.10-8.78(\mathrm{M}, 12 \mathrm{H}, \\
\text { Ar-H) }\end{array}$ \\
\hline
\end{tabular}

Table 4- Antimicrobial screening results of monoazo disperse dyes.

\begin{tabular}{|c|c|c|c|c|c|}
\hline \multirow{3}{*}{ Dye No. } & \multicolumn{5}{|c|}{ Zone of inhibition (mm) } \\
\hline & \multicolumn{4}{|c|}{ Antibacterial activity } & \multirow{2}{*}{$\frac{\text { Antifungal activity }}{\text { C. albicans }}$} \\
\hline & E. coli & P. aeruginosa & S. aureus & B. subtilis & \\
\hline $\mathrm{SP}_{1}$ & 11 & 11 & 10 & 12 & 10 \\
\hline $\mathrm{SP}_{2}$ & 10 & 12 & 13 & 14 & 12 \\
\hline $\mathrm{SP}_{3}$ & 14 & 10 & 19 & 20 & 12 \\
\hline $\mathrm{SP}_{4}$ & 9 & 10 & 14 & 15 & 11 \\
\hline $\mathrm{SP}_{5}$ & 10 & 11 & 10 & 14 & 10 \\
\hline $\mathrm{SP}_{6}$ & 12 & 12 & 14 & 16 & 12 \\
\hline $\mathrm{SP}_{7}$ & 11 & 9 & 12 & 12 & 10 \\
\hline $\mathrm{SP}_{8}$ & 13 & 11 & 18 & 17 & 11 \\
\hline $\mathrm{SP}_{9}$ & 10 & 10 & 11 & 14 & 10 \\
\hline $\mathrm{SP}_{10}$ & 9 & 9 & 12 & 11 & 10 \\
\hline $\mathrm{SP}_{11}$ & 10 & 11 & 13 & 15 & 11 \\
\hline $\mathrm{SP}_{12}$ & 11 & 10 & 13 & 14 & 10 \\
\hline $\mathrm{SP}_{13}$ & 9 & 10 & 15 & 11 & 10 \\
\hline $\mathrm{SP}_{14}$ & 10 & 11 & 14 & 16 & 12 \\
\hline $\mathrm{SP}_{15}$ & 12 & 9 & 12 & 17 & 11 \\
\hline Ciprofloxacin & 19 & 18 & 24 & 28 & - \\
\hline Flucanazole & - & - & - & - & 19 \\
\hline
\end{tabular}

